\title{
Cytological diagnosis of breast tumours by the imprint method
}

\author{
C. R. TRIBE ${ }^{1}$ \\ From the Department of Morbid Anatomy, Stoke Mandeville Hospital, Aylesbury
}

SYNOPSIS Tumour imprints were made from 311 breast tumours at the time of frozen section. Rapid and permanent staining methods were employed and the accuracy of diagnosis was compared with that obtained from frozen and paraffin sections. Two false positives $(0.65 \%)$ and 16 false negatives $(5.15 \%)$ were found in the imprint group compared with no false positives and five false negatives $(1.6 \%)$ in the frozen section group.

The cytology of breast imprints is described and the false results are analysed. The possible uses of this technique, both alone and in combination with frozen sections, are discussed.

As the subject for his Presidential address to the first meeting of the British Society of Clinical Cytology in 1962 Dr. J. Bamforth (1963) chose the cytological diagnosis of tumours. He illustrated his address by a series of beautiful photomicrographs made from the original smears prepared by Professor L. S. Dudgeon in his pioneer work on the cytology of tumours nearly 40 years ago. No one hearing the address could fail to be convinced of the value of this technique. However, in spite of this early work published by Dudgeon in conjunction with Patrick in 1927 and Barrett in 1934, there are surprisingly few references to the use of cytology in the diagnosis of tumours in the British and American literature. This is in marked contrast to European practice where smear techniques, usually based on needle biopsy specimens, have been in use for many years.

The imprint method allows cytological techniques to be used for the examination of individual cells yet preserves to some extent the histological pattern of the imprinted tissue. As a diagnostic method it lies between cytological smear techniques and routine histopathological sections. Its value has already been realized in the diagnosis of diseases of lymph nodes (Moore and Reagan, 1953; Ultmann, Koprowska, and Engle, 1958) and it has been employed by European authors in the diagnosis of breast tumours (Mouriquand and Dargent, 1957; Marsan and Bertini, 1960).

The purpose of this paper is to compare the results

'Present address: Pathology Laboratory, War Memorial Hospital, High Wycombe, Bucks.

Received for publication 17 February 1964. of imprint cytology with both frozen and paraffin methods and to assess its value both as a rapid and a standard method for the diagnosis of breast tumours.

\section{MATERIAL AND METHODS}

In the district served by this department between 1960 and 1963 rapid histological diagnoses by means of frozen sections at the time of operation were made on 311 breast tumours ('lumps in the breast'). At the same time imprints were taken of all these tumours and stained later for a retrospective study. A proportion were also stained by a rapid method and the results compared with those of the simultaneous frozen section.

The method of imprinting is exceedingly simple. The only apparatus needed is a supply of clean microscope slides. After taking the portion of breast tissue required for frozen section an adjacent portion of tissue is trimmed to approximately $\frac{1}{2}$ in. diameter. The tissue is then held between the first finger and thumb so that it protrudes slightly beyond the finger tips and pressed firmly onto a clean microscope slide held in the other hand. It is essential that there should be no lateral or sliding movement. It is then withdrawn, again without sliding, and pressed once or twice more further down the slide. In this manner a series of imprints is obtained similar to pressing a rubber stamp onto paper (Fig. 1). The process can be immediately repeated on several more slides. The slides are then allowed to dry in air and stained by either rapid or routine methods.

The following points should be noted:

1 The tissue surface to be imprinted should be flat and there should be no portions of fat protruding from the edges as these tend to smudge the imprint.

2 Sometimes the first imprint contains excess tissue fluid and blood and it will be found that the subsequent imprints give better cytological results. 
3 The ease with which any one tumour imprints varies considerably. Although malignant tumours of the breast usually imprint more easily than benign lesions, there are exceptions, notably the fibroadenomata. In order to obtain imprints nearest to one cell thick the amount of pressure applied at the time of imprinting therefore varies. With a little experience the macroscopic appearance of the tumours and the appearance of the first imprinted slide allows further imprints to be made with the correct pressure. Benign-looking lesions especially require very firm pressure in order to obtain sufficient cells for diagnosis.

\section{STAINING METHODS}

RAPID Various methods were tried. Some Romanowsky stains will produce a readable result in five minutes but rarely in any shorter period. The best method was found to be that using polychromatic methylene blue in the following manner:

1 Flood the dried imprint with $1 \%$ polychromatic methylene blue for 30 to 60 seconds, depending on the rapidity of the particular batch of stain.

2 Wash with tap water.

3 Mount wet with a coverslip, squashing out the excess air bubbles.

This impermanent rapid method can produce a readable cytological slide within two minutes of imprinting. By using this method a cytological diagnosis can be attempted before looking at the frozen section.

ROUTINE The air-dried imprints were stained with MayGrünwald-Giemsa stain exactly as for blood or bone marrow films. Papanicolau and haematoxylin and eosin stains on wet-fixed imprints were also tried but the Romanowsky-stained films seemed to give better results. This may have been due to the fact that I was used to interpreting the cytology of effusions stained in this manner (Spriggs, 1957). The other methods are not condemned and readers accustomed to a certain cytological staining technique may well prefer their own variant.

\section{RESULTS}

These results are all based on a retrospective study of the permanent May-Grünwald-Giemsa-stained imprints. They were examined with no knowledge of the macroscopic or histological appearances of the tumours concerned. The results were then compared with the diagnoses established from the frozen and paraffin sections.

\section{TABLE I}

COMPARISON OF RESULTS IN DIAGNOSING 311 BREAST TUMOURS BY PARAFFIN AND FROZEN SECTIONS AND IMPRINT CYTOLOGY

Frozen Sections

Imprints

Malignant Benign Malignant Benign

\begin{tabular}{lrrrrr}
\hline $\begin{array}{l}\text { Paraffin sections diagnosed } \\
\text { as malignant 142 }\end{array}$ & 137 & 5 & 126 & 16 \\
$\begin{array}{l}\text { Paraffin sections diagnosed } \\
\text { as benign } \quad 169\end{array}$ & 0 & 169 & 2 & 167
\end{tabular}

DIAGNOSIS OF MALIGNANCY The prime purpose of most cytodiagnostic techniques is to distinguish. between benign and malignant cells. The result $\overrightarrow{\text { F }}$ based on this distinction are shown in Table $I$.

Assuming the paraffin section diagnoses to b를 correct the following false results occurred:

\begin{tabular}{|c|c|c|}
\hline & False Positives & False Negatives \\
\hline $\begin{array}{l}\text { Frozen sections } \\
\text { Imprints }\end{array}$ & $\begin{array}{l}0 \\
2(0.65 \%)\end{array}$ & $\begin{array}{c}5(1.6 \%) \\
16(5 \cdot 15 \%)\end{array}$ \\
\hline
\end{tabular}

It must be appreciated that when interpreting the्यु results of both frozen sections and imprints in this laboratory, if there was any doubt in the pathologist' mind he always inclined to call the lesion benigipo rather than malignant. It is also assumed that the final diagnosis made on paraffin sections was the correct one, though this diagnosis may not necess? arily have been made on the same portion of tissueimprinted or taken for frozen section. Both these factors tend to weigh the results towards false negatives rather than positives.

Frozen sections Five cases diagnosed as malignant on paraffin sections were called benign at time off frozen section though three of them were suspicious ${ }^{\circ}$ of malignancy. It is interesting to note that three out of the five corresponding imprints were correctlys diagnosed as malignant in the retrospective study.

Imprints One of the two cases incorrectlyo diagnosed as malignant proved to be a pericanali- $\frac{\mathbb{Q}}{\Omega}$ cular fibroadenoma. It has been found throughout this study that on imprinting fibroadenomata a3 surprisingly large amount of cellular material is transferred to the slide. This hypercellularity, usually associated with malignant imprints, was a confusing element in the cytological diagnosis off these tumours. However, on microscopy the benign nature of these imprints was usually obvious and they could often be distinguished from the largero number of other benign imprints. Other authors writing on the cytology of breast tumours have also 5 had false positives with fibroadenomata (Dudgeon and Patrick, 1927; Dudgeon and Barrett, 1934; Mouriquand and Dargent, 1957). Since these tumours $N$ have a characteristic macroscopic appearance it is thought that with the use of rapid staining methodso at the time of operation this type of false positivew could be excluded.

The second false positive imprint proved to be ao case of cystic mastitis with areas of apocrineD epitheliosis. On further study of the imprint with $a^{?}$ knowledge of the true diagnosis there still appear to ${ }^{\circ}$ be clumps of malignant cells present.

Sixteen cases which were considered to be cytologically benign were found to be malignant on histological examination. 
It has been the custom in this laboratory for some years to grade breast carcinomata, both at the time of frozen section and subsequently on the paraffin sections, by the method of Bloom and Richardson (1957). Table II compares the final histological grades made on the 126 correctly diagnosed tumours and the 16 false negative results.

\section{TABLE II}

\begin{tabular}{lccc} 
Grade of Cancer & Low & Intermediate & High \\
\hline $\begin{array}{l}\text { False negatives (16) } \\
\begin{array}{l}\text { Carcinomata correctly } \\
\text { diagnosed as malignant by }\end{array}\end{array}$ & 9 & 6 & 1 \\
imprint cytology (126) & 28 & 59 & 39
\end{tabular}

This shows that the false negatives occurred chiefly in the low-grade cancers. The majority of these tumours were scirrhous spheroidal cell carcinomata. Unless particular care is taken when imprinting this type of carcinoma with a dense fibrous stroma too few cells are imprinted for a certain cytological diagnosis of malignancy to be made. Other authors have noted this difficulty with scirrhous carcinomata (Marsan and Bertini, 1960).

It has been found possible to grade the malignant imprints and to obtain results very similar to those made from frozen and paraffin sections. As will be seen in the figures illustrating the cytological criteria, the lower the grade of tumour the more closely the imprinted cells resemble benign epithelial breast cells (compare Fig. 4 with Fig. 9). This factor may lead to false negatives due to lack of clear cytological evidence of malignancy.

On re-examination of these imprints after the true diagnoses were known seven of the 16 cases did show definite malignant cells and their misdiagnosis must be attributed to inexperience and the natural tendency to call doubtful lesions benign. The remaining nine cases can be attributed to faulty technique, since even when the lesions were known to be malignant no definite malignant cells could be seen. Four of these imprints were made from scirrhous low-grade carcinomata and they all showed 'messy' slides with a great excess of red blood cells and tissue fluid.

DIAGNOSIS OF BENIGN LESIONS Of the 169 benign lesions, 126 came into a mixed group including chronic cystic and fibrous mastitis, intraduct papillomata, and gynaecomastia; 35 were fibroadenomata and eight were chronic breast abscesses.

The diagnoses by frozen section on these cases were all in agreement with the final diagnoses by paraffin section.

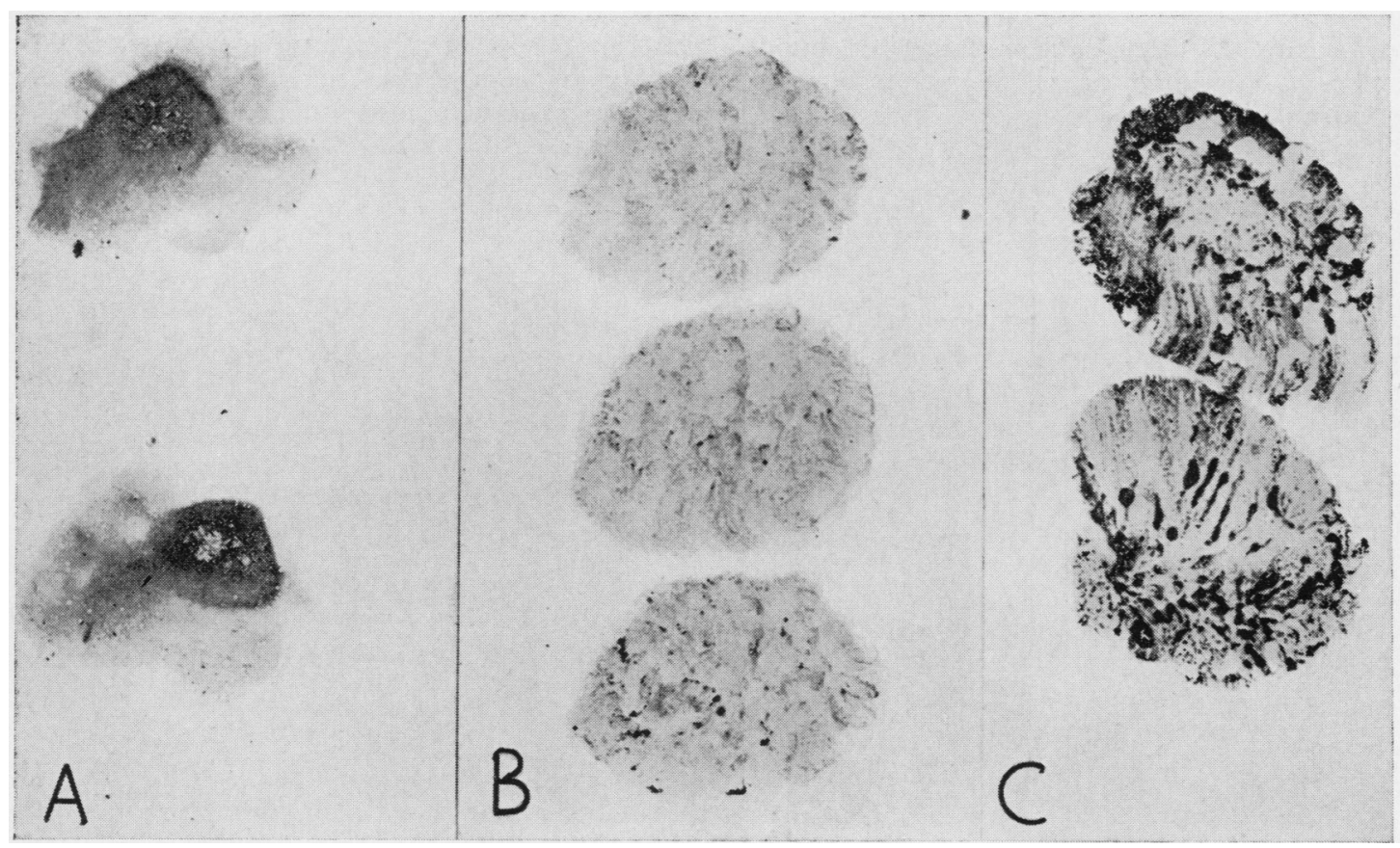

FIG. 1. Naked-eye appearance of different breast tumour imprints. A Mastitis. B Fibroadenoma. C Carcinoma. (approximately $\times 2$ ). 

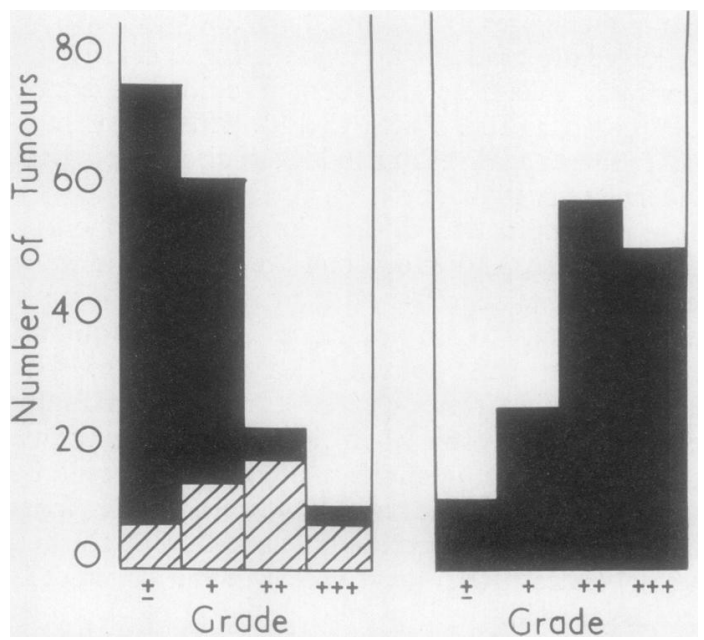

FIG. 2. Histograms showing the difference in grades of cellularity between imprints (left) from 169 benign and (right) 142 malignant tumours of the breast. The hatched portions of the columns in the histogram of benign tumours indicates the proportion of these tumours which were fibroadenomata and breast abscosses.

On examination of the corresponding imprints the eight breast abscesses were successfully distinguished due to the presence of acute and chronic inflammatory cells. The remaining benign lesions were usually indistinguishable, although as mentioned before, a proportion of the fibroadenomata were successfully diagnosed. later.

The cytology of benign lesions will be discuss

LOW-POWER MICROSCOPY Naked-eye examinatiơ of the stained imprint often gives the first clue to the final diagnosis. Some typical examples are shown $\frac{0}{6}$ Figure 1. Low-power microscopy adds further clués and the majority of malignant imprints could distinguished at this stage in examination.

From these two examinations and a knowledg of the histological structure of the equivale tumours, certain basic conclusions were drawn. These were: 1 Malignant tumours imprint mofe easily than benign lesions. 2 The higher the grades of malignancy of any tumour the more easily it witt imprint.

In an attempt to test these conclusions when examining each imprint under low-power micr@્ scopy an estimate was made of the degree cellularity. This was given an arbitrary grading from $\ldots$ via $\therefore$ and . - to - . - .

From an analysis of these grades histograno were prepared (Figs. 2 and 3 ) and these revealed certain basic patterns: (a) As a general rule of imprinting benign lesions of the breast fewer cels were removed than with malignant lesions. (b) The only exception to $(a)$ were the fibroadenomata, the imprints of which were often surprisingly cellula® with many clumps. They comprised more than hat the benign lesions graded ++ and ++ , althoug $B$

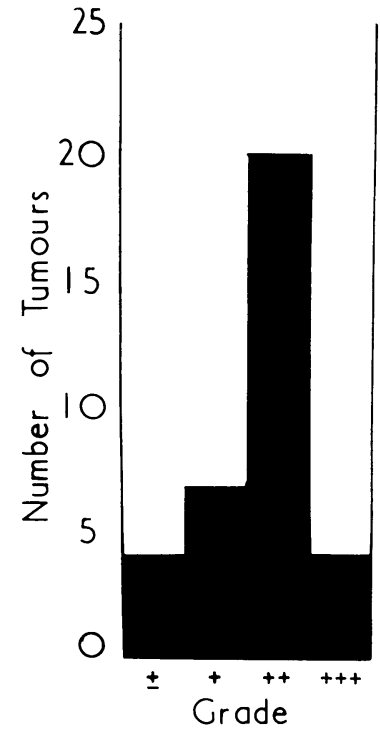

FIG. 3a

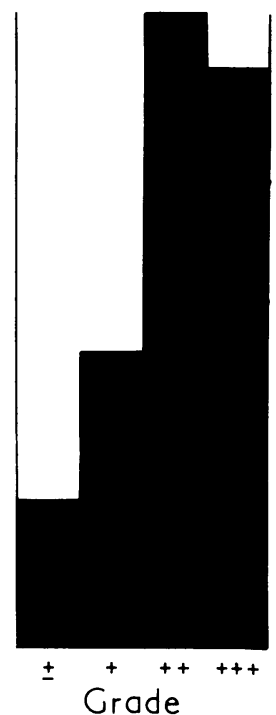

FIG. $3 b$

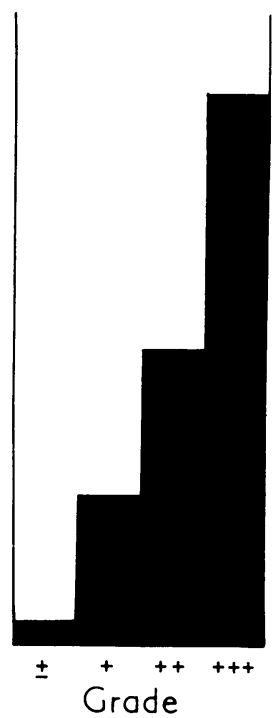

FIG. $3 c$
FIG. 3. Histograms showing the differences in cellularity between imprints from malignant breast tumours of different histological grades. Fig. 3 a shows the degree of cellularity of 35 low-grade tumours, Fig. $3 b$ of 66 of the intermediate grade, and Fig. $3 c$ of 41 high-grade tumours. 
numbering only a fifth of this group. (c) Although not invariable, the more malignant the tumour the more cellular the imprint. This difference was more marked between low and medium grade tumours than between medium and high grades. This is thought to be due to the fact that the majority of scirrhous carcinomata were of a low grade of malignancy.

HIGH-POWER MICROSCOPY This is used to make the final diagnosis, to grade the malignant imprints, and also to study the cytology of the benign lesions.

MALIGNANT CELLS FOUND IN BREAST IMPRINTS These are illustrated in Figs. 4-8 and were identified by the following criteria:

1 Size This is the most important criterion, as with one exception (q.v.) all malignant cells are larger than benign cells. For this reason it is important to use the same magnification when examining imprints by high-power microscopy. As a general rule the larger the cells the higher the grade of malignancy.

2 Pleomorphism In comparison with benign cells, malignant cells all showed some variation in nuclear size and shape. In the higher grade tumours pleomorphism is marked and bizarre giant forms, although not infrequent, are virtually diagnostic if seen (Fig. 6).

3 Nuclear-cytoplasmic ratio This was usually high in malignant cells. Surprisingly in a small proportion of the malignant imprints there was a complete absence of cytoplasm (Fig. 8).

4 Nucleoli These were always more numerous and prominent in the malignant cells. One type of malignant cell had solitary very large blue nucleoli (Fig. 7); more commonly there were multiple small nucleoli.

5 Mitoses These were only infrequently seen. In approximately five minutes' study of each of 126 proven malignant imprints, mitoses were only seen in 20 cases. In the majority of these only one mitosis was seen during this period. However, no mitoses were seen in any of the benign lesions, so if seen they are highly suggestive of malignancy. The reason for this paucity of mitoses in comparison with routine histology is not clear. It is thought that the mitosing cells tend to rupture at the time of imprinting (Fig. 8).

None of these criteria are individually pathognomonic of malignancy. Only by assessing all the features of the imprinted cells can a diagnosis of malignancy be made. Increasing confidence in diagnostic accuracy is gained with experience.

BENIGN CELLS FOUND IN BREAST IMPRINTS Cells found on imprinting benign lesions of the breast

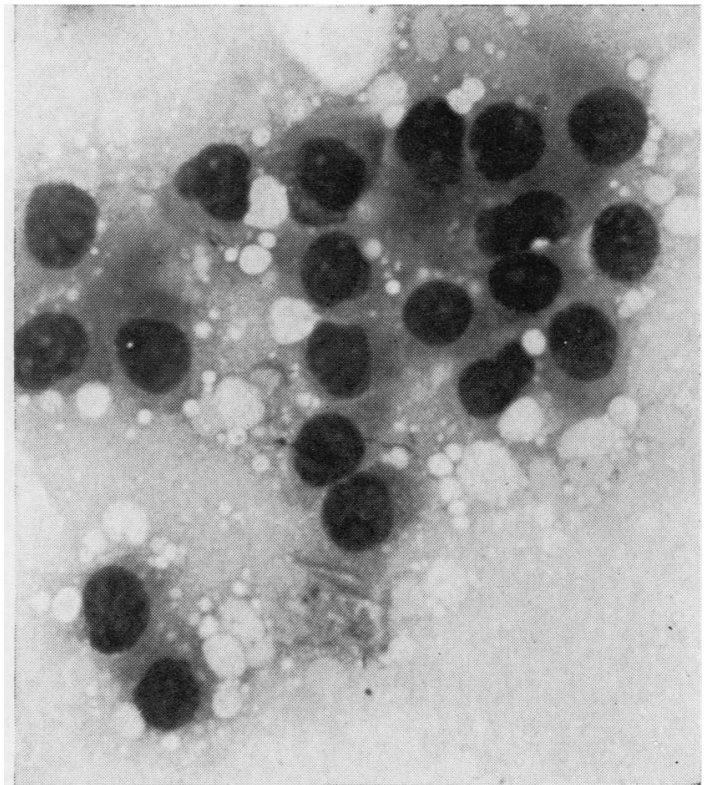

FIG. 4. A clump of tumour cells imprinted from a lowgrade carcinoma of the breast (May-Grünwald-Giemsa $\times 400)$.

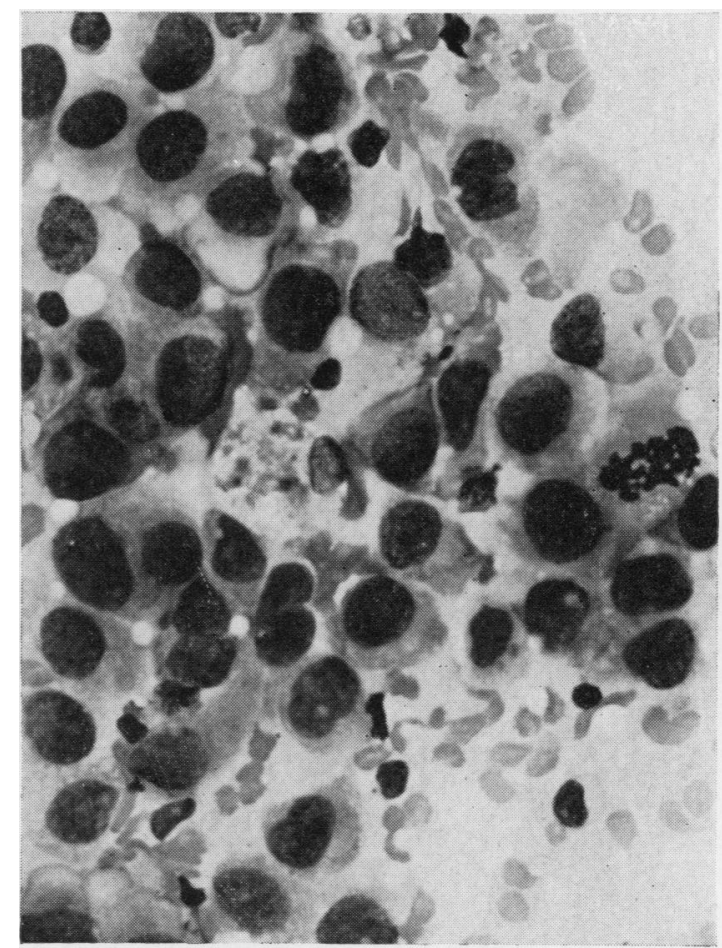

FIG. 5. Imprinted tumour cells from an intermediate grade carcinoma of the breast. Note the mitosis near the right side of the picture (May-Grünwald-Giemsa $\times 400$ ). 


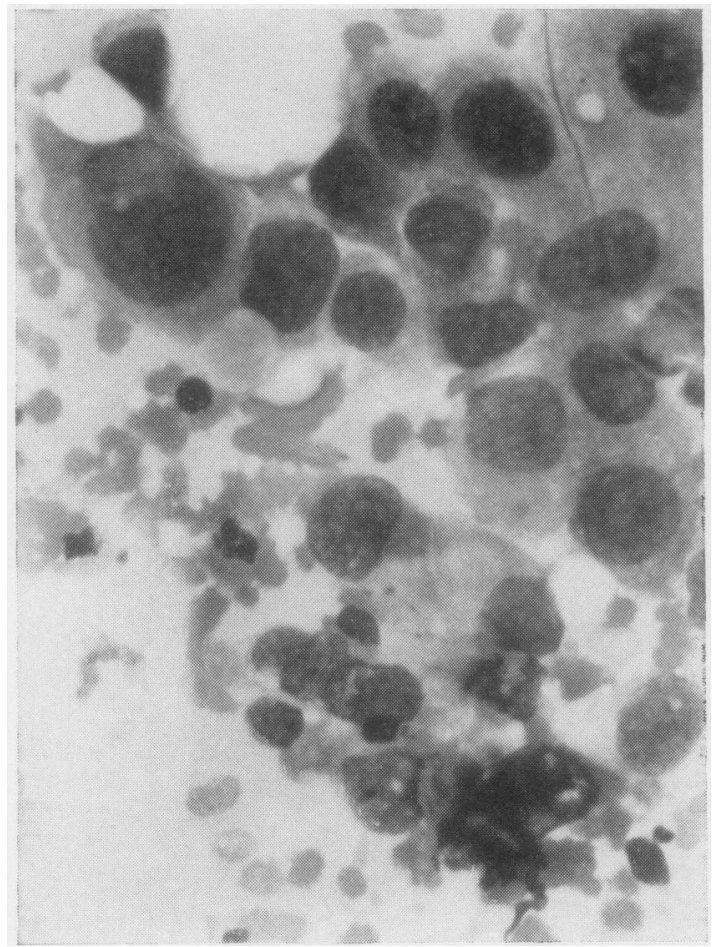

FIG. 6

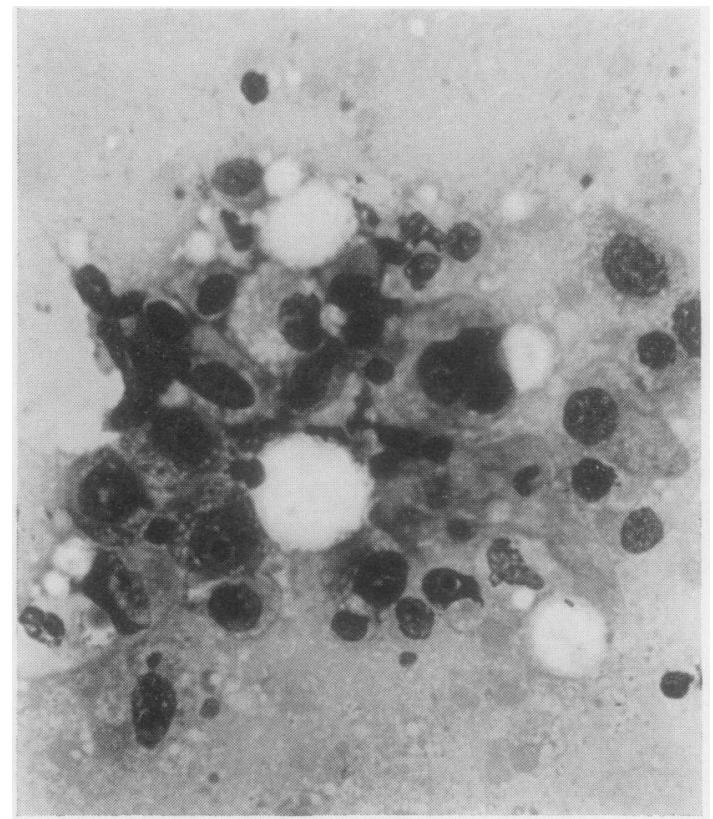

FIG. 7
FIG. 6. Imprinted tumour cells from a high-grå̊ge carcinoma of the breast (May-Grünwald-Giemsa $\times 400) ?$

FIG. 7. Imprinted tumour cells from a high-grate carcinoma of the breast showing large dark blue nucleagi (May-Grünwald-Giemsa $\times$ 400).

ज

FIG. 8. Imprinted tumour cells from an intermediate gradte carcinoma of the breast showing complete lack of cyt plasm and a smeared mitosis (May-Grünwald-Giemo $\times 400)$. 


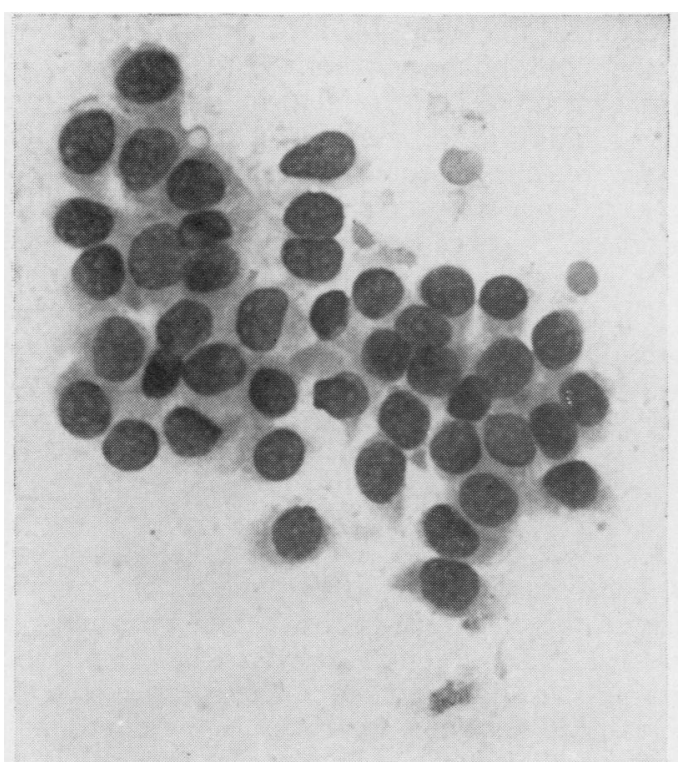

FIG. 9. A clump of benign alveolar and duct lining cells imprinted from a case of cystic mastitis (May-GrünwaldGiemsa $\times$ 400).

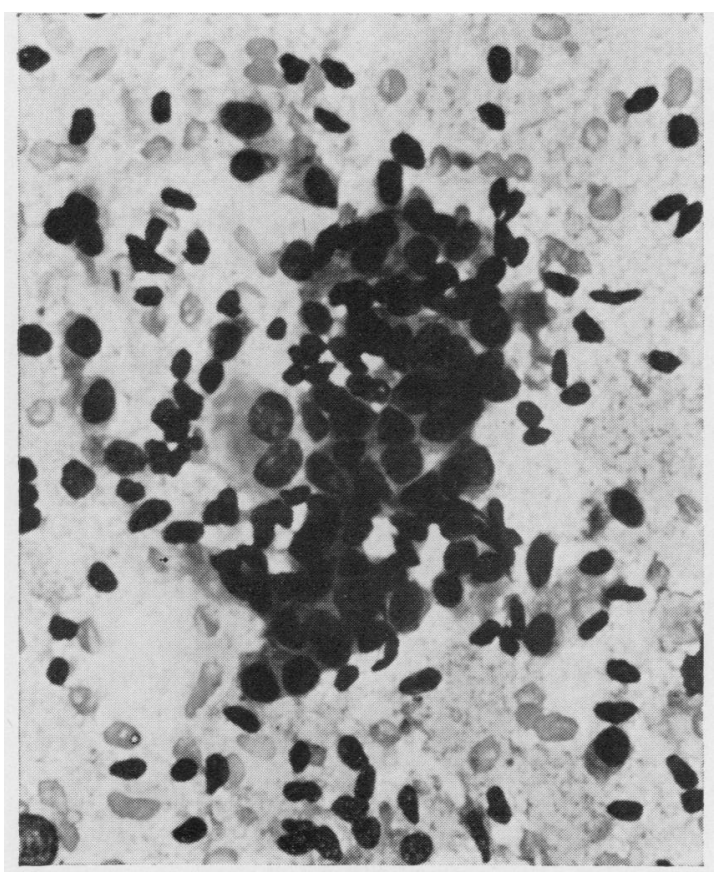

FIG. 10. Benign cells imprinted from a fibroadenoma (May-Grünwald-Giemsa $\times$ 400).

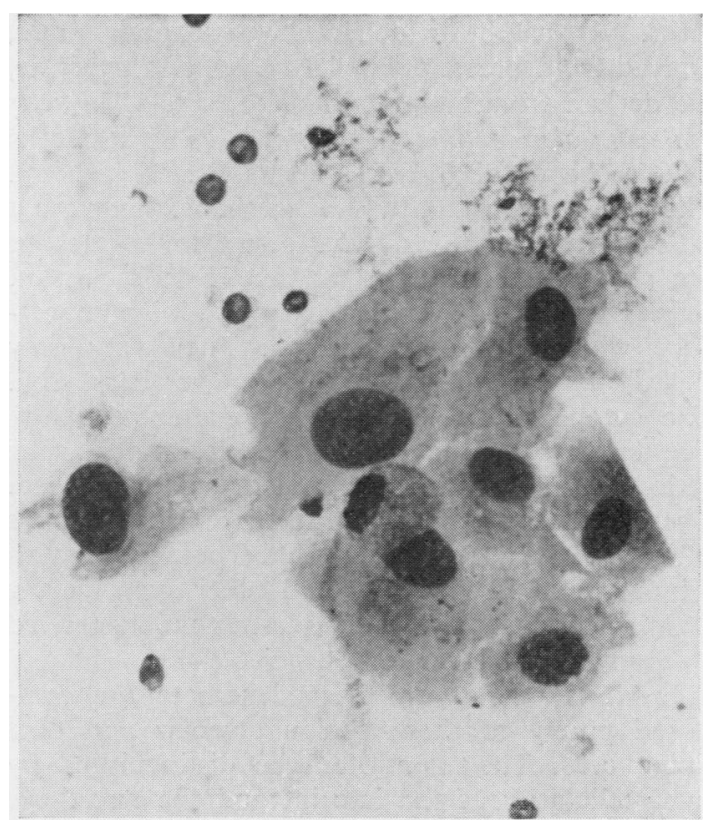

FIG. 11. Apocrine metaplastic cells imprinted from a case of cystic mastitis (May-Grünwald-Giemsa $\times 400$ ).

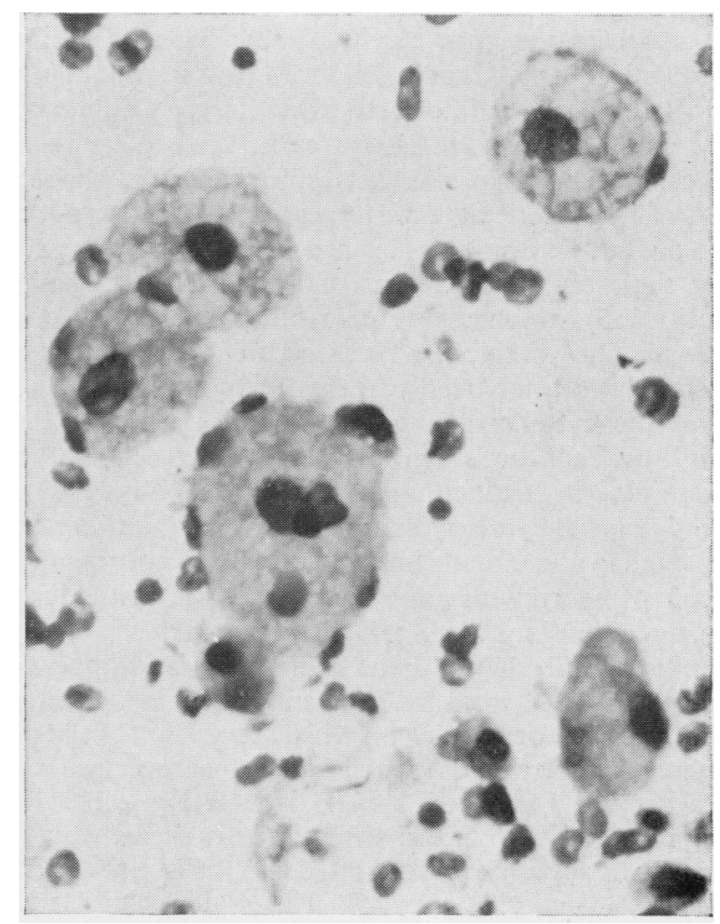

FIG. 12. Foam cells as frequently found in imprints from benign lesions (May-Grünwald-Giemsa $\times$ 400). 
(Figs. 9-12) can be divided into two groups, intrinsic, derived from breast epithelial tissue, and extrinsic, not derived from breast epithelial tissue.

Benign alveolar and duct lining cells These cells have a characteristic appearance (Figs. 9 and 10). The nuclei are round or oval in shape. They are a little larger than the nucleus of a lymphocyte but are always smaller than the nuclei of malignant cells. They have a granular, darkly staining appearance with only small nucleoli. They vary only slightly in size and mitoses have never been seen. The cytoplasm, which is approximately equal in size to that of the nucleus (nuclear-cytoplasmic ratio $1: 1$ ), may be round, oval, or spindle shaped. These cells tend to be imprinted in small clumps often at the edge of the imprint. There is often an admixture of lymphocytes and other small darkly staining cells with scanty cytoplasm possibly derived from myoepithelial cells.

Imprints made from fibroadenomata are composed purely of these benign alveolar and duct lining cells. They can often be distinguished by hypercellularity of the imprint and the naked-eye appearance of the stained slide (Fig. 10).

In hyperplastic benign breast lesions (cystic mastitis, mastopathies) in addition to the cells described above, two other cell types can be distinguished.

Apocrine metaplastic cells These have an easily recognized appearance (Fig. 11) and are the only benign cells which due to their large size might be confused with malignant cells. As can be seen the nuclei vary in size but only slightly in shape; they have a reticulate pattern and usually one dark nucleolus. However, they are instantly recognized by their abundant cytoplasm (nuclear-cytoplasmic ratio $1: 5)$ which stains pale blue. These cells are thought to derive from areas of pink apocrine epitheliosis often seen in cases of chronic mastitis, and the cytoplasm contains granules which stain pink by Lendrum's method (1945). They tend to be imprinted in small clumps and sheets. Beilby (1964), studying the cytology of fluid aspirated from breast cysts, has also noted this type of cell and correlated their presence with apocrine metaplastic epithelium in the equivalent histological material.

Foam cells These have a characteristic appearance (Fig. 12) and are frequently seen in imprints from cases of cystic mastitis. It is not certain whether they represent fat-laden macrophages or degenerate forms of breast duct lining cells. In support of the latter theory, cells intermediate between apocrine cells and foam cells are often seen, i.e., apocrine cells with vacuolated cytoplasm. However, the nuclei of typical foam cells are always much smaller than those of apocrine cells and in appearance they resemble foamy macrophages see in other cytological preparations.

The presence of foam cells indicates some for $\overrightarrow{\overline{7}}$ of benign cystic mastitis and therefore usual indicates a benign condition. This is not invariabe as carcinoma may sometimes coexist with mastiti Foam cells have never been found in imprints made from necrotic degenerating malignant tumours.

The extrinsic cells seen in benign imprints include red blood cells and all types of inflammatory cells If the latter predominate in an imprint they indicate the presence of a breast abscess. Some foam celse are also probably of extrinsic origin.

\section{DISCUSSION}

Before discussing the part that imprint cytology can play in the diagnosis of breast tumours it must b\& stressed that the results described in the previous section were derived from a retrospective study of permanently stained imprints. In addition, in proportion of cases, an imprint was also stained bu the rapid method at the time of the frozen section and examined before the latter. No accurate figures of the results of these rapid imprints were kept as they were usually performed on tumours whict appeared macroscopically malignant and th selection would naturally have biased any suct results. However, it is my firm opinion that accurate cytological diagnoses can be made by this method using the same cytological criteria as those described for the permanent staining method.

In this series the cytological evidence gleaned from prior examination of an imprint not infree quently provided additional diagnostic evidence in case which might otherwise have been reported $\mathrm{ds}$ 'uncertain'. Therefore, although the rapid impring method is advocated as an adequate means of rapig diagnosis of breast tumours on its own, if facilitie for performing frozen sections are available, a combination of the two methods will produce an overall raising of diagnostic accuracy. This combing ation provides something for the pathologist to $d \bar{e}$. while waiting for the frozen section and as hey experience and confidence increases he will be able to diagnose an increasing number of cases fror cytology alone.

Apart from the work of Gibson and Smith (195) there is little reference to the results of cytodiagnosis following needle aspiration of breast tumours in the country. It seems that, rightly or wrongly, surgeons and radiotherapists in this country prefer to have $\$$ histological diagnosis of breast tumours following surgical biopsy before deciding upon the necessars treatment. If this is true, it remains to decides whether rapid cytological methods, either by 
smearing or imprinting the biopsied tumour, can give results comparable to the commonly used frozen section histological technique.

Dudgeon and Barrett (1934), using a rapid smear technique, obtained five false positives and seven false negatives in a series of 212 breast tumours. It is interesting to note that four out of their five false positives occurred in fibroadenomata. Unfortunately cytodiagnosis at this time was in its infancy and this impressive pioneer work was not followed up.

The use of imprints in the diagnosis of breast tumours is described by several French authors. Mouriquand and Dargent (1957), in an article entitled 'L'empreinte mammaire', described their results in a series of 150 cases. Using a rapid Papanicolau method taking only four minutes, they had a $4 \%$ overall error. This consisted of three false positives (two fibroadenomata and one mastopathy) and three false negatives out of 94 carcinomata and 56 benign breast tumours. These figures, whilst agreeing fairly well with those obtained in my series, suggest a slightly less cautious approach to the diagnosis of malignancy. In 1959 Mouriquand reviewed the place of smears from nipple discharges, imprints, and aspiration puncture smears in the cytological examination of breast tumours. She stressed the 'topographical factor' of the imprint method and thought that it merited a place in the rapid examination of breast tumours.

Marsan and Bertini (1960) reviewed the cytological methods used in diagnosing breast tumours. In their small series of 31 breast imprints they had only one incorrect result, a false negative occurring in a very sclerotic tumour with minimal epithelial proliferation.

Castelain and Castelain, in a series of articles in 1951, 1953, and 1955 based on cytological material from 3,000 breast lesions, conclude that rapid cytodiagnosis from smears and imprints has a diagnostic value comparable with rapid histological methods. They used May-Grünwald-Giesma stains and stress the simplicity of the technique, even suggesting that its use could make an important economy in the national budget'. In another article in 1956 these authors used similar techniques in examining lymph node metastases and state that such examination allows 'prognostic interpretation' and 'determination of sensitivity to physical agents of any one tumour'. In a few cases I have also examined the axillary lymph nodes from fresh mastectomy specimens by imprints and found the identification of metastases relatively easy.
How do these cytological results compare with those obtained from frozen sections? The latter technique is in more common use in America than in Great Britain, and Sparkman (1962) analysed the reliability of frozen sections in the diagnosis of breast lesions in seven recent American papers from 1957 to 1961 . Out of a total 3,536 cases there were $61(1.7 \%)$ deferred diagnoses, 22 false negatives $(0.6 \%)$, and no false positives. The five false negatives and no false positives in the present series of 311 cases are comparable with the above figures,

It is apparent, both from the results quoted from previous authors and from those obtained in the present series, that smear or imprint cytological diagnostic methods used by themselves are not quite as reliable as frozen sections. However, as shown in the analysis of false results, it is thought that the results obtained in this series could be considerably improved by more meticulous technique and with further experience in the interpretation of imprint cytology.

In conclusion is seems that tumour imprints will probably never replace first class frozen sections, especially those produced on cold microtomes of the Cryostat type. It is suggested, however, that they have a definite place used in combination with frozen sections. Also in countries where there is a lack of trained technicians or apparatus the diagnosis of breast tumours can be made with a high degree of accuracy solely from imprint cytology.

I wish to thank Dr. H. J. Harris for his encouragement and histological aid in this series. I am also grateful to Dr. A. I. Spriggs and Dr. M. Wolfendale for their advice and criticism, to Mrs. W. Boast for technical assistance, and to Miss Kenya Smith for the histograms and secretarial aid.

\section{REFERENCES}

Bamforth, J. (1963). J. clin. Path., 16, 395.

Beilby, J. O. W. (1964). Personal communication.

Bloom, H. J. G., and Richardson, W. W. (1957). Brit. J. Cancer, 11, 359.

Castelain, G., and Castelain, C. (1951). Presse méd., 59, 1783.

- - (1953). Ibid., 61, 1020. (1955). Ibid., 63, 764. (1956). Ibid., 64, 2204.

Dudgeon, L. S., and Barrett, N. R. (1934). Brit. J. Surg., 22, 4.

-, and Patrick, C. V. (1927). Ibid., 15, 250.

Gibson, A., and Smith, G. (1957). Ibid., 45, 236.

Lendrum, A. C. (1945). J. Path. Bact., 57, 267.

Marsan, C., and Bertini, B. (1960). Path. et Biol., 8, 343.

Moore, R. D., and Reagan, J. W. (1953). Cancer (Philad.), 6, 606. Mouriquand, J. (1959). Rev. franc. Gynéc., 54, 427.

- , and Dargent, M. (1957). Bull. Ass. franc. Cancer, 44, 449.

Sparkman, R. S. (1962). Ann. Surg., 155, 924.

Spriggs, A. I. (1957). The Cytology of Effusions. Heinemann, London. Ultmann, J. E., Koprowska, I., and Engle, R. L. Jr. (1958). Cancer Philad.), 11, 507. 